\title{
Multiple cerebral venous sinus thrombosis: Case report
}

\begin{abstract}
E. Mogere, MBChB, Resident, Department of Surgery, The Aga Khan University Hospital, Nairobi, Kenya and D.O. Olunya, FRCS (Neurosurg), Consultant Neurosurgeon and Senior Lecturer, Department of Surgery, The Aga Khan University Hospital, P.O. Box 30270-00100, Nairobi, Kenya

Correspondence and reprint requests to:

Dr. E. Mogere, Department of Surgery, The Aga Khan University Hospital, P.O. Box 30270-00100, Nairobi, Kenya, Email: edwin.mogere@akhskenya.org
\end{abstract}

\section{Summary}

Cerebral venous thrombosis (CVT) is an uncommon clinical problem and can be characterised by nonspecific and common symptoms of headaches and vomiting due to the intracranial hypertension. Alternate diagnoses are entertained especially when procoagulant factors are not elicited. We present a 35 year old female, parity $2+0$, gravida 3 at 8 weeks gestation who presented with headache, vomiting, photophobia and neck stiffness. The diagnostic workup and radiological findings confirmed multiple venous sinus thromboses. The case is discussed in the light of diagnostic challenge, treatment pathways and how an understanding of the basic sciences explains its clinical presentation.

The diagnosis of cerebral venous sinus thrombosis should be considered in a young patient presenting with an unusual headache in the absence the usual vascular risk factors.

\section{Introduction}

Cerebral venous thrombosis is an uncommon disease that presents in a non-specific pattern. Ninety percent of patients report a headache, $50 \%$ have some type of neurological deficit and convulsions occur in $40 \%$ of cases (1). This nonspecific presentation leads to misdiagnosis, delayed diagnosis or medical misadventures. Average delay from onset of symptoms to diagnosis is 7 days (2).
The patient profile is varied, defining clinical characteristics few and aetiological relationships diverse. Documented series indicate association of sinus thrombosis with presence of prothromotic factors (3), genetic factors including protein $\mathrm{C}$ and $\mathrm{S}$ deficiency, pregnancy, nephrotic syndrome as well as dehydration and cancer (4).

A clinician's high index of suspicion remains the vanguard of making an accurate and timely diagnosis. We report a case of a young female who presented with non-specific symptoms and signs and managed for multiple cerebral venous sinus thrombosis.

\section{Case report}

A. N., a 35 year old female patient, at parity $2+0$, gravida 3 at 8 weeks gestation presented to our hospital with 6 days history of a severe global throbbing headache of sudden onset that was associated with vomiting, photophobia and neck stiffness. She had no antecedent trauma, fever nor any history of hypertension.

Her obstetric and gynecological history was unremarkable, without any use of oral contraceptives. She was a married housewife who neither smoked nor consumed any alcohol.

On general examination, A.N. was restless but had no conjuctival pallor, scleral icterus, oral thrush or lymphadenopathy. Her vital signs were normal (brachial blood pressure of $106 /{ }_{65} \mathrm{mmHg}$, temperature $37^{\circ} \mathrm{C}$ ) except for a 
tachycardia of 106 beats/min (good volume, regular non-collapsing pulse).

She was fully conscious with normal higher functions and pupils that were $3 \mathrm{~mm}$ in diameter and responsive to light, both directly and consensually. Positive findings included photophobia, a left sided abducent nerve palsy and bilateral papilloedema at fundoscopy. Her neck was stiff with negative Kerning's and Brudzinki's signs while the spine, motor, sensory and cerebellar examinations were unremarkable. The systemic findings were noncontributory. An impression of a subarachnoid hemorrhage with differential diagnosis of meningitis and an intracranial space occupying lesion were entertained.
Laboratory investigations included a haemogram $\left(\mathrm{Hb}\right.$ of $13.7 \mathrm{~g} / \mathrm{dL}, \mathrm{WBC}$ of $8.2 \times 10^{9}$ $/ \mathrm{L}$ with a neutrophil count of $70 \%$ ) and electrolytes $(\mathrm{K}+2.8 \mathrm{mmol} / \mathrm{L})$. The rest of the biochemistry, and the coagulation profile were unremarkable. The opening pressures were high and the CSF bloody at lumbar puncture.

An MRI was then performed that revealed hyperintense signals in the superior sagittal sinus (SSS), straight, right transverse and sigmoid sinuses-changes due to thrombosis (Figure 1). The thrombi were multiple with some being contiguous and others discrete.

A thrombophilia screen ordered after this showed a normal profile (Table 1).

Table 1: Thrombophilia screen for A.N.

\begin{tabular}{lcc} 
& Level & Ref: \\
Antithrombin III & $106 \%$ & $(80-120 \%)$ \\
Protein C activity & $120 \%$ & $(69-124 \%)$ \\
Protein S activity & $63 \%$ & $(62-145 \%)$ \\
Homocysteine level & $7.1 \mu \mathrm{mol} / \mathrm{L}$ & $(3.4-20)$ \\
Antiphospholipid antibodies & Negative & \\
\hline
\end{tabular}

Figure 1: T1 MRI images of the patient showing hyperintense signals at multiple sites (block arrows)

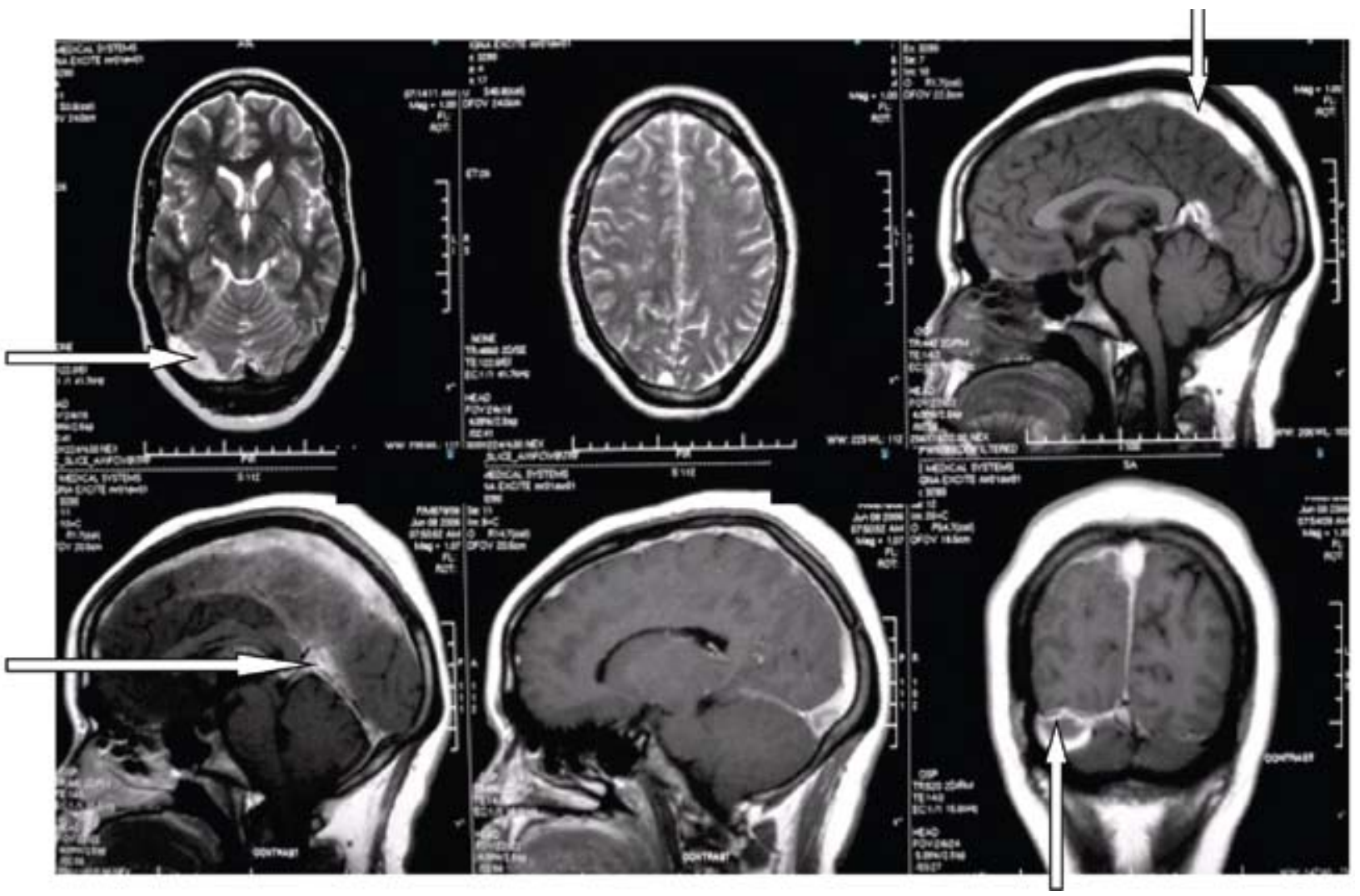


Her subsequent management entailed anticoagulation with high dose enoxaparin and treatment with clonazepam, analgesics and potassium supplementation. The patient had an uneventful recovery and was discharged home on the $7^{\text {th }}$ day. She will complete one year of anticoagulation.

\section{Discussion}

Most of A.N.'s major venous sinuses were involved with the exception of the inferior saggital. The superior saggittal sinus usually drains into the confluence of sinuses then into the straight and finally the sigmoid sinus. The combination of both continuous and discrete thrombi point to either a systemic aetiology or a combination of both local and systemic causes. Some anatomic features may explain some of the local factors contributing to thrombosis and propagation. Sinuses are valveless endothelialined channels formed between the two layers of the dura. Endothelial disruptions in these low pressure channels (contributing to stasis) may initiate or propagate thrombosis (5). The cerebral veins join the sinuses obliquely generally against the blood stream and create regions of turbulent flow. These features may point to prothrombotic zones and explain thrombus propagation in sinuses in proximity. Hence a systemic hypercoagulable state in combination with local features may account for the varied pattern of thromboses.
This patient had no other procoagulant factor other than pregnancy. High circulating estrogen levels in pregnancy are accepted to be the cause of this hypercoagulable state. In general, aetiological relationships of cerebral sinus thrombosis are varied. About $85 \%$ of patients are noted to have a procoagulant factor (3) which may include protein $C$ and $S$ genetic deficiencies, pregnancy, nephrotic syndrome as well as dehydration and cancer (4). Her age and physiological status did not fall into the other thrombophilia risk patterns.

Close to $90 \%$ of patients report a headache with $50 \%$ having some type of neurological deficit while convulsions occur in $40 \%$ of cases (1). Headache was present in our patient. This symptom, in association with vomiting is a feature of intracranial hypertension. Normal cerebro-spinal fluid flow patterns have the final common pathway as the reabsorption by the arachnoid granulations and flow into the venous sinuses (6). Thrombosis within the sinuses block the CSF flow pathways causing intracranial hypertension without ventriculomegaly (Figure 1). Cerebral vein thrombosis leads to local effects of venous hypertension with associated ischemia and disruption in the blood brain barrier that result in both cytotoxic and vasogenic edema (Figure 2), which contribute further to the development of intracranial hypertension. These distinct but concurrent mechanisms are central to the pathophysiology. Large unilateral lesions may cause compression of the diencephalon and brain stem leading to coma or death.

Figure 1: Description of the course and consequence of venous thrombosis

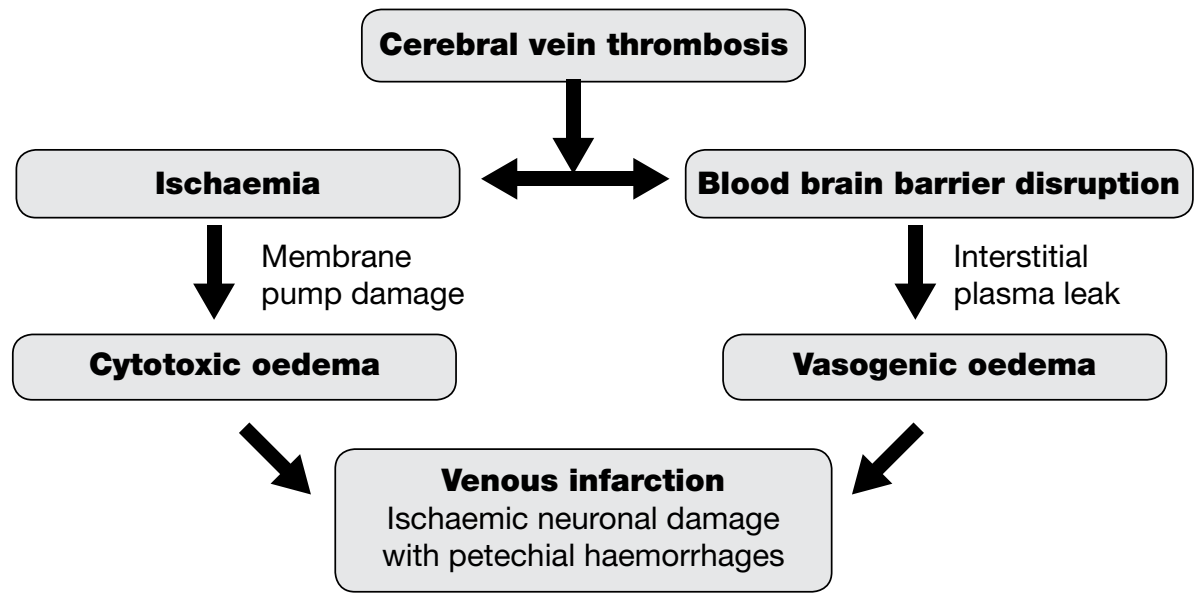


Figure 2: Effects of major sinus thrombosis

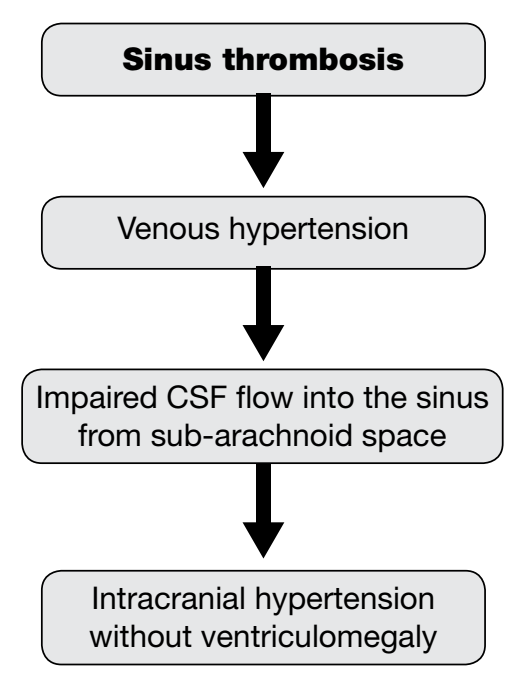

The clinical features in our patient varied with limited value in terms of localization of the lesion. She had features of meningeal irritation and abducent nerve palsy. Since the sinus drainage areas are wide and varied, the neurological signs seen here and described by other authors are not surprising.

The diagnosis of CVT is usually confirmed on laboratory investigations and radiology. The lumbar puncture was performed and revealed bloody CSF. This is a sensitive but nonspecific marker for cerebral venous thrombosis. The fact that our patient was pregnant limited our radiological investigation to the MRI since a CT scan poses unacceptable radiation risks to the fetus. In routine practice the sensitivity of CT scan is limited in the initial three days but its utility lies in the ability to rapidly rule out other important differential diagnoses. MRI approaches a sensitivity of up to $98 \%$ in some series with the current modality of choice being Single-slice phase-contrast angiography (SSPCA) with a sensitivity of $100 \%$ (7).

Treatment of CVT is aimed at stabilization, prevention of clot propagation and the prevention or reversal of cerebral herniation. The management of this patient included anticoagulation for the purposes of propagation prevention. This role of anticoagulants is controversial with the basic concern being that $40 \%$ of patients present with a haemorrhagic infarct in situ. Literature available from three trials by Einhaupal et al (8), De bruin et al (9) and Nagaraja et al (10) provide no clear answers. In all these, no increase or new cerebral haemorrhage occurred. Two cases of pulmonary embolism were reported in the placebo group in two these trials.

The duration of anticoagulation is guided by the findings that there is a $2 \%$ rethrombosis and $4 \%$ extracranial thrombotic event within a year (1). This formed the basis for one year of anticoagulation in our patient.

The clinical course of the patient was benign with self-limiting disease without need for surgery to achieve decompression or use of osmotic diuretics (11), as well as endovascular placement of thrombolytics (12). The latter is considered experimental. Although the patient had a lumbar puncture for diagnostic purposes, it could also be therapeutic for the treatment of intracranial hypertension. If employed, the punctures are done serially in combination with acetazolamide (13). CSF diversionary procedures are indicated after two weeks of failed conservative therapy with serial lumbar punctures. The options include lumbar peritoneal shunt or fenestration of the optic nerve sheath (14).

In conclusion, this patient's non-specific clinical picture belies the challenge in diagnosis and management of this particular condition. Although a rare condition, a clinician's high index of suspicion remains the first step towards a correct and timely diagnosis. The diagnosis of cerebral venous sinus thrombosis should be considered in a young and middle-aged patient presenting with an unusual headache or with stroke-like symptoms without the usual vascular risk factors. The worsening of the local effect of cerebral venous thrombosis is preventable by early anticoagulation.

\section{Acknowledgements}

I am indebted to my two teachers Dr. Saidi Hassan and Dr. Abdulkarim Abdallah for their constant encouragement and support. I am grateful to Millicent Mayienga of the Department of Surgery, Aga Khan University 
Hospital, Nairobi for her input in preparing the script as well as Prof. Asad Raja for his unwavering faith and patience to all his charges, myself included.

\section{References}

1. De Bruijn S.F., Stam J. and Kappelle L.J. Thunderclap headache as first symptom of cerebral venous sinus thrombosis. Lancet 1996; 348: 1623-1625.

2. Ferro J.M., Canhao P., Stam J., et al. Prognosis of cerebral vein and dural sinus thrombosis: results of the International Study on Cerebral Vein and Dural Sinus Thrombosis (ISCVT). Stroke 2004; 35: 664-670.

3. Stam J. CVS Thrombosis. New England J. Med. 2005; 352; 17: 1791-1798.

4. Deschiens M.A., Conard J., Horellou M.H., et al. Coagulation studies, factor V Leiden, \& anticardiolipin antibodies in 40 cases of cerebral venous thrombosis. Stroke 1996; 27: 1724-1730.

5. Browder J. and Kaplan H.A. Cerebral Dural Sinuses and their Tributaries. Springfield, Illinois, 1976.

6. Kida S., Weller R.O. Morphology of CSF drainage pathways in man. Principles of Pediatric Neurosurgery, vol 4. Berlin: Springer. 1994.

7. Adams W.M., Laitt R.D., Beards S.C., et al. Use SSPCA for the diagnosis of dural venous sinus thrombosis. Eur Radiol. 1999; 9(8): 1614-1619.
8. Einhaupl K.M., Villringer A., Meister W., et al. Heparin treatment in sinus venous thrombosis. Lancet. 1991; 338: 597-600.

9. de Bruijn S.F. and Stam J. Randomized, placebocontrolled trial of anticoagulant treatment with lowmolecular-weight heparin for cerebral sinus thrombosis. Stroke. 1999; 30: 484-488.

10. Nagaraja D., Rao B.S.S., Taly A.B., et al. Randomized controlled trial of heparin in puerperal cerebral venous/sinus thrombosis. Nimhans J. 1995; 13: 111-115.

11. Stefini R., Latronico N., Cornali C., et al. Emergent decompressive craniectomy in patients with fixed dilated pupils due to cerebral venous and dural sinus thrombosis: report of three cases. Neurosurgery. 1999; 45: 626-629.

12. Canhão P., Falcao F. and Ferro J.M. Thrombolytics for cerebral sinus thrombosis: A systematic review. Cerebrovasc Dis. 2003; 15: 159-166.

13. Kesler A., Hadayer A., Goldhammer Y., Almog Y., et al. Idiopathic intracranial hypertension: risk of recurrences. Neurology. 2004; 63: 1737-1739.

14. Tse D.T. and Chang W.J. Surgery of the orbit and optic nerve. In: Glaser J.S., editor Neuroophthalmology. 3rd ed. Philadelphia, Lippincott Williams \& Wilkins, 1999; 520-523. 